\title{
Life Post Bariatric Surgery: The Experiences of People with Type 2 Diabetes Mellitus: A Qualitative Study
}

\author{
Alanoud M Alobaidly ${ }^{1}$, Wendy Abigail ${ }^{2}$ and Pauline Hill ${ }^{3}$ \\ ${ }^{1}$ College of Nursing \& Health Sciences, Flinders University, South Australia \\ ${ }^{2}$ College of Nursing \& Health Sciences, Flinders University, South Australia \\ ${ }^{3}$ College of Nursing \& Health Sciences, Flinders University, South Australia
}

Submission: April 18, 2020; Published: May 22, 2020

*Corresponding author: Alanoud Alobaidly, College of Nursing and Health Sciences, Flinders University, Sturt Road, Bedford Park 5042, South Australia,

\section{Abstract}

Introduction: Kuwait ranks 8th in the prevalence of obesity and 9th in diabetes mellitus worldwide. Recently, physicians have introduced bariatric surgery as a treatment option for people with type 2 diabetes mellitus.

Purpose: This study aimed to understand the experiences of Kuwaitis with type 2 diabetes mellitus after bariatric surgery on their diabetes self-management and daily routine.

Methods: Using Stake's (2005) instrumental case study methodology; email interviews with four Kuwaiti participants diagnosed with type 2 diabetes mellitus at six to 12 months after bariatric surgery was conducted The data was analysed by following Creswell's [1] six steps to thematic analysis and Thomas [2] quality and positionality to increase the rigour of the study.

Results: 'Knowledge', 'Satisfaction from results', and 'Healthcare management' were the three major themes identified from the interviews. These themes revealed an absence of patient education for bariatric surgery, unsuccessful diabetes education for people with type 2 diabetes mellitus, and the lack of a multidisciplinary team approach in managing people with type 2 diabetes mellitus and bariatric surgery.

Conclusions: The collaboration of specialist physicians and surgeons is significant for the benefit of patient's health. This research can assist in creating suitable patient education programmes and improving health care practices for this population.

Keywords: Life experiences; Patient education; Type 2 diabetes mellitus; Bariatric surgery; Obesity

\section{Introduction}

Kuwait provides a comfortable life for its citizens after the discovery of oil in 1938 [3] in which they have adapted an inactive lifestyle; high-fat low fiber diets; and received high incomes contributing to the rise of obesity, associated comorbidities and non-communicable diseases including, cardiovascular diseases and type 2 diabetes mellitus (T2DM) [4,5]. As a consequence, Kuwait ranked the 8th highest country worldwide for the prevalence of obesity and 9th in diabetes in 2017 [6,7]. The prevalence of diabetes in Kuwait is at $23 \%$ where $14.3 \%$ were adults aged 20-79 years, making it one of the top 10 leading countries in the world for diabetes prevalence [3]. Bariatric surgery (BS) was a treatment for obesity in Kuwait, where almost $80 \%$ of its population were considered overweight and $50 \%$ obese [3]. Literature has shown the rewarding outcomes of managing blood glucose levels (BGLs) within normal ranges and achieving target weight loss results for the population who were overweight, obese and morbidly obese by undergoing BS in [8]. BS has been shown to have promising results on weight loss and reduction of comorbidities that has led to increased quality of life [9]. The literature suggested that lifestyle changes and education should be maintained to prolong the health and sustainability of the positive outcomes of BS. Treating bariatric patients psychologically as well as physiologically aids in maintaining a healthy life [10]. Doing so would significantly increase the quality of life for people with T2DM who have undergone BS and aid in positive long-term results from the surgery. Psychological support has been reported as beneficial for the bariatric population in delaying negative health outcomes [11]. However, professional 


\section{Current Research in Diabetes \& Obesity Journal}

psychological support has been avoided by Kuwaiti individuals due to cultural factors like stigma, family ties, gossip and soiled reputations [12]. This might have influenced the support that bariatric people seek to address concerns regarding undergoing this surgical intervention.

Overall, there is a lack of knowledge concerning the physical and psychological health of the Kuwaiti population undergoing BS as a treatment option for T2DM. Research is required to narrow this gap. Therefore, the aim of this research was to answer the question: "What are the experiences of people with T2DM after BS in Kuwait?". The research question focuses on people with T2DM in Kuwait at six to 12 months after BS, which has been reported as showing promising results in several health aspects including body weight and BGL. To explore the effects of BS in relation to T2DM in a Kuwaiti culture, three sub-questions were considered: 1. What were the experiences of people with T2DM in Kuwait after BS?; 2. What were the effects of BS on the diabetes management of people with T2DM?; 3 . What were the types of support that this population received pre and post BS? The purpose of this project was to contribute to the body of knowledge in this emerging area of health care by gaining understanding and insight into the

research questions (Table 1). phenomenon.

\section{Material and Methods}

Stakes' (2005) [2] instrumental case study methodology was implemented to gain insight and advance understanding features from real-life events of people with T2DM in Kuwait six to 12 months after BS that could illustrate what is occurring and reasons behind this occurrence. Ethical approval was granted by the with Social and Behavioural Research Ethics Committee at Flinders University (approval number 7730) in South Australia and by the Hospital Director and Head of Surgical Department in Kuwait.

\section{Settings and sample}

A convenience sampling strategy was chosen due to its accessibility and efficiency [13]. Participants were recruited from a bariatric clinic at one of the government hospitals in Kuwait. The clinic receptionists recruited patients who met the criteria (T2DM and at six to 12 months post BS) where they were handed the information sheet and asked to contact the researcher to participate in the study. For this case study research, four volunteers participated and provided rich data to answer the

Table 1: Demographic data.

\begin{tabular}{|c|c|c|c|c|}
\hline Participants & Participant \#1 & Participant \#2 & Participant \#3 & Participant \#4 \\
\hline Age & $19-34$ & $35-50$ & $19-34$ & Over 50 \\
\hline Gender & Male & Male & Female & Female \\
\hline Marital Status & Married & Divorced & Single & Kuwaiti \\
\hline Nationality & Kuwaiti & Kuwaiti & Kuwaiti & Unemployed \\
\hline Employment Status & Employed & Unemployed & 5 years & Over 10 years \\
\hline Duration of T2DM & 1 year & 7 years & 125 & 124 \\
\hline Weight before BS & 142 & 165 & 61 & 65 \\
\hline Present weight & 72 & 75 & 8 months & 11 months \\
\hline Period of BS & 6 months & 12 months & & \\
\hline
\end{tabular}

\begin{tabular}{|c|c|c|}
\hline Type of BS & RYBG & 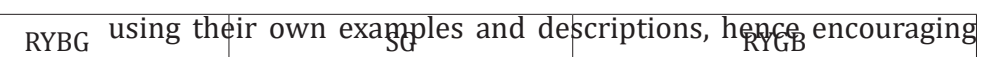 \\
\hline
\end{tabular}

\section{Data collection}

Semi-structured email interviews were conducted to encourage participants to communicate freely and comfortably a greater depth of insight into the phenomena [13]. Through email interviewing, participants were not pressurized to provide answers promptly, giving them more time to reflect on their responses [14]. The interview questions were developed based

on the literature reviewed that investigated the effects of BS on participants' social, personal and work life (Table 2).

Table 2: Interview questions.

\begin{tabular}{|c|}
\hline Q1: When did you find out that you have had type 2 diabetes mellitus? \\
\hline What was the reason for your diabetes? \\
\hline Do you keep track of your blood sugar [glucose]? [If no, what were the barriers you faced?] \\
\hline *Was your blood glucose levels checked during these visits to the hospital? If it was, do you know was the results were? How often do you check it? \\
\hline Q2: Describe to me how you felt the first time you checked your weight after surgery? \\
\hline How much weight have you lost until now? \\
\hline
\end{tabular}




\section{Current Research in Diabetes \& Obesity Journal}

\begin{tabular}{|c|}
\hline What type of bariatric surgery did you have? \\
\hline Q3: What helped your decision to have bariatric surgery? \\
\hline Q4: What has changed in your eating habits after surgery [if any]? \\
\hline Q5: What changes did you feel in your physical activity after surgery [if any]? \\
\hline *Could you tell me about how you feel when doing everyday tasks? \\
\hline Q6: What were the changes in your diabetes medication after surgery [if any]? \\
\hline Q7: Wescribe to me how your life has changed since you had the surgery? \\
\hline Q8: Did you receive support before or after surgery? \\
\hline Who supported you? \\
\hline How did they support you? \\
\hline Q9: What do you suppose is the reason people would want to have this surgery? \\
\hline * Were health care professionals [dietician, physiotherapist, and psychologist] available for needed support? [if yes, how did they support you?] \\
\hline ** Could you please explain why you have chosen to hide the fact of going through this lifechanging surgery? \\
\hline Q* What do you think of people treating their health issues with this type of surgery instead of the conventional way of exercise and a balanced diet? \\
\hline
\end{tabular}

$\left.{ }^{*}\right) 2^{\text {nd }}$ Interview clarification for respondents and follow up questions, $\left({ }^{* *}\right) 3^{\text {rd }}$ Interview follow up questions.

\section{Results}

Creswell's [1] six steps of thematic data analysis was used to analyse the data Thomas' [2] positionality and quality were used to provide rigour for the interpreted data. Three main themes were identified: knowledge, satisfaction from results, and healthcare management. Each theme generated subthemes that explained the experiences of this population.

\section{Theme 1: Knowledge}

Participants were asked about their routine care of their T2DM, what reasons they believed were behind their T2DM, what they knew about BS and whether there were any changes in their knowledge following the surgery. The three subthemes identified are 'management of Type 2 diabetes mellitus', 'awareness of nutritional needs' and 'belief in bariatric surgery as a cure for T2DM'.

Subtheme 1: Management of T2DM: The interviews revealed that most of the participants had limited knowledge and management of their T2DM. Participant 1 who had been recently diagnosed, often replied in a negative manner by rejecting and expressing their indifference in managing their T2DM when they said:

“I don't check it (BGL). It's (BGL) only check when I visit the hospital for routine check-ups, which is not often, thank god... I don't really remember the results." - Participant 1 Limited knowledge on their condition was revealed when they said:

“The nurse said it's a bit high but they didn't give me anything for it (high BGL)." - Participant 1
According to Participant 2 and 3, their knowledge on T2DM was limited. Although they adhered to their hypoglycaemic medication regimes, they did not follow the recommended regime of monitoring their BGL due to the physical discomfort of pricking their finger. They reported:

“No (I don't check it). It's (BGL checking) painful and I don't think it's too high... (I check my BGL) when I feel tired or I go to the toilet frequently" - Participant 2

"Yes (I keep track of my BGLs), (I check my BGLs) twice a week and when I don't feel normal... I used to take Glucophage" - Participant 3

Participant 4 was more active with their T2DM management than the other participants and had adopted BGL monitoring into their daily routine. This participant stated:

"Yes, almost every day (I check my BGLs), in the morning before breakfast and at night before I go to sleep" - Participant 4

Subtheme 2: Awareness of nutritional needs: The participants reported that their meal portions, frequency and quality [type] of food had changed and that they were aware of the different food groups and which to avoid. They also reported craving certain foods due to the high caloric intake of fat and sugar in the participants diet pre-surgery and in the short time they possessed to adjust their portion sizes. For example, one participant said:

"I have become aware of how my body works and what my body needs... I have reduced my sugar, fats, carbohydrates and salt... (I eat more) Chocolate and chips"." - Participant 3

Another aspect explored was participants' knowledge on their nutritional habits and other factors that might have contributed to their T2DM. Participants all agreed that their food, exercise 
and/or heavy weight played a significant role in their diagnosis of T2DM, for example; one participant said:

"I think it's because of my lifestyle... I don't like going to the gym... I love to eat food rich in flavour and fats. I prefer sitting at the café with friends" - Participant 1

\section{Subtheme 3: Belief in bariatric surgery as a 'cure' for T2DM}

Participants' limited knowledge on BS and the influence of the doctors performing the surgery led them to believe that BS could 'cure' them of T2DM in addition to reducing their weight. For example, one participant said:

"The fastest treatment for diabetes and overweight (is BS) the doctor told me that the surgery can cure my diabetes... I don't have diabetes anymore 8 months after surgery." Participant 2

Bariatric surgery also helped the participants with other obesity related co-morbidities such as sleep apnoea and hypertension, which were the several reasons participants provided for undergoing the surgery. Although the participants had limited knowledge on the outcomes of the surgery, their beliefs of the benefits on their health seemed to be predominately their doctors' opinion and advice, including the encouragement to undergo BS to be 'cure' from the chronic health condition. Participants said:

"Other than my diabetes, I was diagnosed with high blood pressure so I made the decision (to have BS)... (After BS) I was surprised that my diabetes was gone. The doctor told me that I might be cured and that I don't need to take diabetes medication anymore" - Participant 1

"(I had BS because) I need to lose weight due to legs and knee pain and difficulty in walking... my doctor encouraged me (to have the surgery)... (After BS) I don't take them anymore (diabetes medication)." - Participant 4

\section{Theme 2: Satisfaction from the results}

Participants were asked about the changes they noticed following the surgery. The two subthemes that emerged and which described the experiences of the participants within this period are: 'physical condition' and 'psychological reaction'.

Subtheme 1: Physical condition: Participants shared their experiences from losing weight [until the day of the interview] and noted the impact of their weight loss on their work environment. Participants said:

"I was assigned to office work before surgery, now I can work as a field worker" - Participant 2

"I felt so excited because I could see the changes in my body... I am also more focused on my work especially because my work is all about numbers." - Participant 3
Changes in participants' physical health were expressed as being their satisfaction from improvement in their level of activity and ease in completing daily tasks. A lighter weight helped one of the participants in spending their family time actively. This provided the participant with a sense of satisfaction from the change in their physical state. Participants were also satisfied after the BS relieved breathing conditions and improved their general health.

Subtheme 2: Psychological reaction: Positive emotions were expressed by the participants upon observing the changes in their body and weighing themselves for the first time after BS, which confirmed their satisfaction with the results of the surgery. Improved confidence after the surgery was also a positive reaction that most of the participants reported. Participants expressed their feelings of satisfaction through their ability to wear nice clothes, while others were confident because they could move easily. Additionally, positive reactions from family and friends who provided support and encouragement throughout the post bariatric experience was discussed by participants. For example, one participant stated:

"My children and my doctor... they stood by me and encouraged me to do the surgery" - Participant 4

One satisfied participant reported a positive reaction with support and encouragement post BS from their family and friends. This participant stated:

"My parents, ex-wife (now), and friends... they always consider the type of food we eat or help me prepare food. The also join and encourage me for sports" - Participant 2

One of the participants did not change their lifestyle habits after weight loss. Although the participant had a positive reaction from the weight loss, when asked about what had changed in their life after surgery, they responded negatively:

“Nothing much changed... It (the surgery) didn't affect my work, I work in an office. It (the surgery) didn't affect my daily routine... I still don't go to the gym" - Participant 1

Fear was another negative reaction experienced by participants. Fear of weight regain was represented in the changes participants made in their diet by consuming low carbohydrate and fat food which were consistent with weight loss diet. Participants stated:

"(I avoid) food that contains high sodium, high fat and high sugar" - Participant 2

"(I avoid) rice, fried food, bread and pastries." - Participant 3

Fear of resentment from others was expressed by one of the participants who kept knowledge of their surgery private because they did not want to be judged by others. This participant stated: 


\section{Current Research in Diabetes \& Obesity Journal}

"Nobody knew about it (BS) because everyone knew I was against this surgery. I've even tried to talk people out of it" Participant 1

Fear of dying due to accumulated comorbidities was also expressed by one of the participants who stated:

"When I got diagnosed with high blood pressure too, and the doctor prescribed tons of pills, I changed my mind (about the surgery) because I got scared." - Participant 1

Another participant reported a negative reaction from their family before surgery. They reported that they did not receive any type of support from their family and were judged and looked at negatively by them for being obese. Participant 3 said:

“The people didn't accept the way I look, especially my family for being fat." - Participant 3

\section{Theme 3: Healthcare management}

This theme aids in understanding the process that the participants experienced before and after BS, and the actual care that the participants were offered. Two subthemes emerged, which were 'individualised care' and 'standardised care'.

Subtheme 1: Individualised care: All participants were treated at the same healthcare organisation, but the management of their BS was different. Three participants were managed by their physician or surgeon and one participant was managed by a team that consisted of a physician and dietitian. Participants said that their physician was the sole support for them during their treatment progress. However, only one participant mentioned the presence of a dietician along with their physician stating:

"My doctor advised me on how to maintain my weight and asks me to continue my follow-ups and dietician gave me with a flexible meal plan" - Participant 2

Subtheme 2: Standardised care: Standardised care provided were follow-up appointments at one, three, six and twelve months, then yearly to keep record of their progression. This type of care was offered for people with T2DM after BS and was similar for all participants. However, there were no reports by the participants on patient education or any organised multidisciplinary team collaboration. Participant 1 said that he was just told what to do even when their BGL was high, where Participant 1 said:

"[The nurse] asked me to keep taking my diabetes medication." - Participant 1

None of the participants reported their awareness of a specific treatment plan or support prior to their surgery but the participants did report scheduling of follow-up appointments.

\section{Discussion}

The medical management of T2DM internationally has focused on managing people's BGLs by providing this population with patient education regarding the effects of diet and exercise on BGLs. However, adapting to changes in diet, physical activity and medication is difficult to maintain, thus often resulting in poor management of T2DM [15]. These factors were considered the conservative method of T2DM management by the International Diabetes Federation (IDF) [15]. Alarming increases of diabetes in Kuwait were contributed to by suboptimal health care, poor diabetes self-management and the lack of diabetes patient education [16]. The current study has identified that knowledge, satisfaction from the results and healthcare management are important areas for people with T2DM following BS. These are discussed in detail as follows.

\section{Knowledge}

Limited knowledge regarding T2DM management, nutritional needs and BS is an issue that leads people with T2DM to undergo BS surgery. The lack of knowledge might also contribute to their uncontrolled BGLs prior to the surgery [17]. Participants in this study were not provided with any patient education for T2DM or BS prior to or following their surgery. This corresponds with the findings of Awad et al. [18] who reported a lack of patient education in T2DM management and Alfadhli et al. [19] who also reported a gap between the health practices and beliefs of a Kuwaiti population and the recommendations for optimal health.

Providing diabetes education to patients improves glycosylated haemoglobin levels in Kuwaiti people [17]. This current study found that limited knowledge was one of the issues that these Kuwaiti people with T2DM had when dealing with their diabetes, which was similar to the findings of Waheedi et al. [17]. This current study also found that monitoring BGLs pre and post BS was challenging due to the discomfort of finger pricking, where most participants were pleased to no longer need to test for BGLs following their BS. Most participants also reported their adherence to their oral hypoglycaemic medications and their unwillingness to monitoring their BGLs, which could expose them to the risk of going into hypoglycaemia or diabetic coma [15]. Seeking surgical options as a cure to T2DM in a population that have received insufficient self-management education seemed ' $f i t^{\prime}$ with the rise of BS in the past decades.

Participants in this study gained nutritional knowledge following BS, which they perceived as contributing to changes in their portion sizes and nutritional intake and eventually assisted in their weight reduction. This finding was similar to the findings of Taube-Schiff et al. [20] when interviewing their participants found the retention of participants' nutritional knowledge following surgery. A decrease in food intake, lower appetite, malabsorption of calories are some effects of BS [21-23]. In this current study, all the Kuwaiti participants also reported their awareness and increased knowledge about healthy eating, which made them avoid certain foods that could contribute to weight gain, such as food rich in carbohydrates and fats. However, most of the Kuwaiti participants stated that they developed cravings of high fat or high 
caloric food such as chocolate and baked chips. Cravings for high fat and high sugary food might be associated with pre-surgery caloric intake where participants have not adjusted to their new weight post surgery. Gaining awareness of foods that are low in glycaemic index might also explain the reasons why participants would choose certain types of food that help in prolonging the rise of their BGLs. Hillersdal et al. [24] had similar outcomes with their participants' eating habits after BS.

All participants in this current study believed that they were 'cured' from T2DM, which gave them a sense of relief from the burden of managing their BGLs. Non-adherence to a diet plan and lack of exercise are factors that challenge people with uncontrolled T2DM [24]. This study found that BS was used as a method to manage obesity and therefore assist with T2DM management when Kuwaiti patients no longer responded to the medications prescribed or they sought an easier and faster option to manage their T2DM. Participants also revealed their beliefs and limited knowledge regarding BS was influenced by their physician. No literature has reported the effect of knowledge on BS as a treatment option on the decision of people with T2DM to have BS, but there are indications that remission of T2DM was one of the outcomes of BS $[23,25,26]$. Although T2DM symptoms were relieved after BS, patients still need to be monitored and managed the same as for people with T2DM. This opposes the findings in this study where the physician and participants believe they were 'cured' from T2DM and no longer required T2DM management.

Although BS might provide people with T2DM the potential to live without symptoms of diabetes and not require management for a brief period, this state is only temporary due to the decrease in weight [27]. Therefore, although people with T2DM appear asymptomatic post-BS, they are expected to develop symptoms again. For example, Himpens et al. [28] reported that their study sample resumed oral hypoglycaemic medication and were re-diagnosed with T2DM nine years post-BS. Ritter et al. [29] suggested that T2DM remission depended on patients' lifestyle modification following the surgery.

\section{Satisfaction from the results}

Physical changes are common in people who have undergone BS. Participants in this study reported that they had a sedentary lifestyle, which presented them with challenges around weight management and activities of daily living, such as difficulties in getting into and out of cars or getting up from a seat. A sedentary lifestyle had led the participants to become obese, which had also contributed to their unhealthy eating habits and decrease in physical activity [3]. The increased incidences of cardiovascular diseases, T2DM and obesity were associated with low levels of exercise in Kuwaiti adults [2], which aligns with the participants interviews that were conducted in this research that explored the experiences of people with T2DM after BS.
Positive outcomes of the physical changes included advances in the some of the participants' careers following T2DM remission that was due to improvements in their body image, level of activity and T2DM management. Maximum weight loss following BS occurred in the first one to two years of surgery [26]. Jumbe et al. [8] reported their participants had positive outcomes from the surgery on their health, appearance, social life and self-esteem. Participants in the current study reported improvements in their breathing conditions such as breathlessness during activity, sleep apnoea and asthma, which were relieved following their surgery. BS positively affected overall health of their participants including hypertension, diabetes, cholesterol and sleep apnoea [26]. Participants in this study also reported negative physical changes such as feeling weaker after losing weight which is consistent with a loss of physical energy in response to a sudden reduction in food intake [21].

Psychological reactions are also common in people post BS. Different psychological reactions were identified regarding the experiences of the participants. One participant said that they had their family's support after BS, and that this support encouraged them to change their lifestyle to maintain weight loss. This corresponds with the findings of who reported that strong family ties in the Kuwaiti culture might affect a decision of an individual to carry out a task. Participants expressed positive emotions after weight loss where they stated they were happy and satisfied from the outcomes of the surgery. Happiness, contentment and satisfaction with weight loss were commonly expressed by people post BS which also improved their self-esteem [10].

Overall, the current study found positive reports from the Kuwaiti participants following BS. However, negative reports were also expressed by participants. These included negative responses from family members, and fear of weight regain. The fear of weight gain was present in the participants of this study despite them demonstrating higher nutritional knowledge regarding long-term dietary requirements. Fear of weight regain was usually seen in people who are obese and have lost significant amount of weight from BS [21]. However, Taube-Schiff et al. [20] stated that nutritional knowledge had no significant impact on their participants' weight loss.

Fear of dying due to accumulated comorbidities was another negative emotion found in this study. This might have also contributed to participants' negative experiences of having the surgery. Jumbe et al. [10] reported the possibility of weight regain in their participants and the need for psychological support to aid with their psychological health throughout the bariatric experience to manage negative experiences and emotions. Participants experienced lack of family support following BS which affected the participants psychologically. Some participants kept their surgery private, which might have been due to their family and friends' disapproval of the BS. Another participant refused 
support from their family after being teased and unaccepted for being obese. These undesired reactions by family members and friends highlighted the impact of social pressure on individuals' psychological health. Jumbe et al. [8] discussed the importance of family support for people undergoing BS to achieve positive outcomes for a longer period.

\section{Healthcare Management}

Standard care was provided by the healthcare facility involved in this study. However, none of the participants were aware of a plan of care after the surgery and were only told to attend followup appointments to maintain their weight. The participants experienced diverse individualised care from the same healthcare facility. A multidisciplinary team including a nutritionist is essential to maintain the favourable outcomes of BS and avoid weight regain following BS [21,30]. This study found a diversity in the care service provided to patients and insufficient patient education.

Patient education for T2DM is practiced in Kuwait through institutions such as Dasman Diabetes Institute. However, there is a gap in this practice provided at the Kuwaiti Health Care Service where only basic T2DM education was provided. Waheedi et al. [17] reported low commitment to diabetes education by the Kuwaiti people, which is due to the limited availability and accessibility of diabetes education. This might explain the reason why none of the participants reported the availability of patient education before their surgery. Diabetes patient education was unsuccessful among the Kuwaiti population due to the lack of cultural adaptation of T2DM self-management programmes for the Kuwaiti culture suggesting a tailored programme for more successful and acceptable approach by a Kuwaiti population with T2DM [16]. Standard care was provided to people with T2DM after BS but there was an absence of a formal multidisciplinary team and patient education in T2DM and BS. This study concurs with Al Slamah et al. [16] and recommends that cultural adoption of Western educational programmes is a necessity for health care providers to be able to successfully educate their patients. This study recommends that a multidisciplinary team is needed in the management of patients with T2DM undergoing BS patients to improve and prolong surgical outcomes.

\section{Limitations}

Several limitations were identified in this research project. This research was conducted using email interviews as the data collection method due to it becoming a modern means of interviewing for researchers conducting research overseas in a limited timeframe; however, it was described as unpopular by the Kuwaiti participants and suggested that telephone interviewing was more practical. Feedback from the participants indicated that they preferred instant messaging or even phone calls, even though they supported research and the need to raise awareness, especially regarding patient education and patients' needs. This study had a small sample size; hence the results cannot be generalised to whole population groups. Despite these limitations, conducting a case study methodology offered insight into the many issues that people undergoing BS might experience which can be used as a reference when planning patient education programmes for a Kuwaiti population of T2DM or BS.

\section{Conclusion}

People in Kuwait rely highly on their doctors' opinion especially when it concerns their health. However, limited knowledge and the inefficient methods of delivering patient education and raising awareness for people seeking surgical options as a treatment would be the reason for this trusting act between the patient and their physicians. Moreover, the impact of the cultural and societal impressions has been significant in the participants of this study. Therefore, the need to provide efficient patient education and the importance of a collaborative approach in the management of this population is necessary to provide efficient delivery of care services. Future directions in research would expanding the population to obtain generalizable findings, and to assess the impact of a multidisciplinary team approach when caring for people with T2DM and BS. It is also important to involve health care managers, stakeholders and policy makers to improve health care services that is most suitable for the Kuwaiti population.

\section{Acknowledgements}

This study was supported by Jaber Al Ahmed Armed Forces Hospital and Civil Services Commission in Kuwait via Higher Degree Research program at Flinders University.

\section{References}

1. Creswell JW (2013) Qualitative Inquiry and Research Design: Choosing among five approaches. ( $3^{\text {rd }}$ Edn.), Thousand Oaks, California, USA: Sage publications.

2. Thomas G (2016) How to do your case study: Sage.

3. Haskin O (2013) Bariatrics in Kuwait: Dr Salman Al Sabah bariatricnews.net.

4. Al-Baho AK, Al-Naar A, Al-Shuaib H, Panicker JK, Gaber S (2016) Levels of physical activity among Kuwaiti adults and perceived barriers. Open Public Health Journal 9(1): 77-87.

5. Zaghloul S, Al-Hooti SN, Al-Hamad N, Al-Zenki S, Alomirah H, et al. (2013) Evidence for nutrition transition in Kuwait: over-consumption of macronutrients and obesity. Public health nutrition 16(04): 596607.

6. World Health Organisation (WHO) (2018) Diabetes Factsheet 2013.

7. World Health Organisation (WHO) (2017) 10 Facts on Obesity WHO int 2016.

8. AlMarri F, Al Sabah S, Al Haddad E, Vaz J (2017) A call for more research from the Arabian Gulf. Obesity Surgery 1-10.

9. Alobaidly AM, Hasan AY, Abigail W, Hill P (2018) A Review of Literature on the experiences of people with Type 2 Diabetes Mellitus after Bariatric Surgery in Kuwait. Kuwait Medical Journal 50(4): 387-398. 
10. Jumbe S, Hamlet C, Meyrick J (2017) Psychological aspects of bariatric surgery as a treatment for obesity. Current Obesity Reports 6(1): 7178.

11. Tarrant M, Khan SS, Farrow CV, Shah P, Daly M, et al. (2017) Patient experiences of a bariatric group programme for managing obesity: A qualitative interview study. Br J Health Psychol 22(1): 77-93.

12. Scull NC, Khullar N, Al-Awadhi N, Erheim R (2014) A qualitative study of the perceptions of mental health care in Kuwait. International Perspectives in Psychology: Research, Practice, Consultation 3(4): 284-299.

13. Polit D, Beck C (2017) Nursing Research Generating and Assessing Evidence for Nursing Practice. (10 ${ }^{\text {th }}$ Edn.), Wolters Kluwer.

14. Hawkins JE, Chard R, Chenail R, Seibert D (2017) The email interview in qualitative research: A discussion of the practical utility. The Qualitative Report Conference.

15. International Diabetes Federation (IDF) (2017) What is diabetes? Facts and Figures IDF.org.

16. Al Slamah T, Nicholl B, Alslail FY, Melville C (2017) Self-management of type 2 diabetes in gulf cooperation council countries: A systematic review. PLoS One 12(12): e0189160.

17. Waheedi M, Awad A, Hatoum H, Enlund H (2017) The relationship between patients' knowledge of diabetes therapeutic goals and selfmanagement behaviour, including adherence. Int J Clin Pharm 39(1) 45-51.

18. Awad A, Dalle H, Enlund H (2011) Diabetic Patients' Knowledge of Therapeutic Goals in Kuwait. Med Princ Pract 20(2): 118-123.

19. Alfadhli S, Al-Mazeedi S, Bodner ME, Dean E (2017) Discordance between Lifestyle-Related Health Practices and Beliefs of People Living in Kuwait: A Community-Based Study. Med Princ Pract 26(1): 10-16.

20. Taube-Schiff M, Yufe S, Dettmer E, D’Agostino NM, Sockalingam S (2016) Bridging the Gap: Patient Experiences Following Transfer of Care from a Pediatric Obesity Management Program to an Adult Bariatric Surgery Program. Bariatr Surg pract Patient Care 11(2): 67-72.

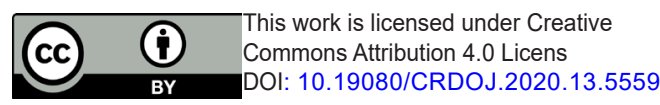

21. Garduno-Diaz SD (2016) Diet-Related Behaviour Modification Following Bariatric Surgery J Am Diet Assoc 3(4): 667-669.

22. Gautier T, Sarcher T, Contival N, Le Roux Y, Alves A (2013) Indications and mid-term results of conversion from sleeve gastrectomy to Rouxen-Y gastric bypass. Obesity Surgery 23(2): 212-215.

23. Jamal M, Aminian A (2015) Bariatric surgery: is it a safe treatment modality? Kuwait Medical Journal 47(3): 193-200.

24. Hillersdal L, Christensen BJ, Holm L (2016) Patients' strategies for eating after gastric bypass surgery: A qualitative study. Eur J Clin Nutr $70(4): 523-527$.

25. Al-Sabah S, Al-Mullav A, Al-Enezi K, Al-Bastaki W, Jumaa T (2013) Laparoscopic Sleeve Gastrectomy for Type 2 Diabetes Mellitus: 4 Years' Experience from Kuwait. Obesity Surgery: Springer 233 Spring St, New York, NY 10013 USA.

26. Mayer JE, Dwyer JT (2016) Bariatric Surgery or Conventional Medical Therapy? Which Is Best for Severely Obese Adults with Type 2 Diabetes? Nutrition Today 51(5): 233-241.

27. Schlienger JL, Pradignac A, Luca F, Meyer L, Rohr S (2009) Medical management of diabetes after bariatric surgery. Diabetes and Metabolism 35(6): 558-561.

28. Himpens J, Verbrugghe A, Cadiere GB, Everaerts W, Greve JW (2012) Long-term results of laparoscopic Roux-en-Y Gastric bypass: evaluation after 9 years. Obesity Surgery 22(10): 1586-1593.

29. Ritter S, Vetter ML, Sarwer DB (2012) Lifestyle modifications and surgical options in the treatment of patients with obesity and type 2 diabetes mellitus. Postgraduate medicine 124(4): 168-180.

30. Al Haqan AA, Al-Taweel DM, Awad A, Wake DJ (2017) Pharmacists' attitudes and role in diabetes management in Kuwait. Medical Principles and Practice 26(3): 273-279.

Your next submission with Juniper Publishers
will reach you the below assets
- Quality Editorial service
- Swift Peer Review
- Reprints availability
- E-prints Service
- Manuscript Podcast for convenient understanding
- Global attainment for your research
- Manuscript accessibility in different formats
( Pdf, E-pub, Full Text, Audio)
- Unceasing customer service
Track the below URL for one-step submission
https://juniperpublishers.com/online-submission.php

